\title{
Long non-coding RNA SUMO1P3 promotes tumour progression by regulating cell proliferation and invasion in glioma
}

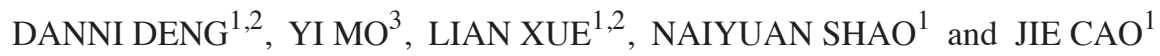 \\ ${ }^{1}$ Department of Neurosurgery; ${ }^{2}$ Clinical Medical Research Center, The First People's Hospital of Changzhou; \\ ${ }^{3}$ Department of Neurosurgery, The Third Affiliated Hospital of Soochow University, Changzhou, Jiangsu 213000, P.R. China
}

Received August 12, 2020; Accepted February 11, 2021

DOI: $10.3892 /$ etm.2021.9922

\begin{abstract}
Gliomas account for $50 \%$ of primary brain tumours in the central nervous system. Small ubiquitin-like modifier 1 pseudogene 3 (SUMO1P3), a newly identified long non-coding RNA (lncRNA), serves an oncogenic role in various types of cancer. The aim of the present study was to investigate the effect of SUMO1P3 on glioma progression. The results demonstrated that SUMO1P3 expression was upregulated in glioma tissues and cell lines. Furthermore, SUMO1P3 was associated with a poor overall survival of patients with glioma. The results of the in vitro cell proliferation and flow cytometry assays demonstrated that SUMO1P3-knockdown suppressed cell proliferation and cell cycle. The results of the wound healing and Transwell assays demonstrated that SUMO1P3-knockdown significantly repressed cell migration and invasion. In addition, SUMO1P3 promoted glioma by regulating the expression levels of $\beta$-catenin, cyclin-D1, N-cadherin and E-cadherin. Overall, the results of the present study suggested that SUMO1P3 may act as an oncogene by regulating cell proliferation, cell cycle, cell migration and invasion in glioma, and may represent a novel diagnostic biomarker and therapeutic target for glioma.
\end{abstract}

\section{Introduction}

Gliomas account for $50 \%$ of primary brain tumours in the central nervous system (1). According to the malignancy of the tumour, the World Health Organization (WHO) categorizes glioma into four grades, I-IV (2). Patients incipiently remain asymptomatic until obvious clinical symptoms appear, such as headaches, seizures, nausea, sensory loss and aphasia (3). Standard treatment of glioma includes surgical excision followed by adjuvant chemotherapy and/or radiotherapy; however, the overall survival of patients diagnosed with malignant glioma

Correspondence to: Dr Jie Cao, Department of Neurosurgery, The First People's Hospital of Changzhou, 185 Juqian Road, Changzhou, Jiangsu 213000, P.R. China

E-mail: mayday509@163.com

Key words: small ubiquitin-like modifier 1 pseudogene 3, glioma, proliferation, cell cycle, migration is poor due to a high risk of relapse $(4,5)$. Thus, an improved understanding of the precise molecular mechanisms underlying glioma pathogenesis is crucial to help provide novel therapeutic candidates for the treatment of glioma.

Long non-coding RNAs (lncRNAs), which are transcripts of $>200$ nucleotides without protein-coding ability, have been demonstrated to serve an essential role in tumour initiation and progression (6). For example, small nucleolar RNA host gene 12 acts as a tumour promoter by regulating cell proliferation, apoptosis, migration and multidrug resistance in gastric, colorectal, non-small cell lung and triple-negative breast cancer (7-10). Metastasis associated lung adenocarcinoma transcript 1 acts as an oncogene in different types of cancer, such as kidney carcinoma, ovarian cancer and colorectal cancer, by regulating cell viability, apoptosis, invasion, migration and epithelial-to-mesenchymal transition (EMT) (11-13). Recently, several studies have revealed that lncRNAs can affect the initiation and progression of glioma (10-12). For example, lncRNA H19 decreases chemoresistance to temozolomide by suppressing EMT via regulation of the Wnt/ $\beta$-catenin signalling pathway in glioma cell lines (14). IncRNA X-inactive specific transcript promotes tumorigenesis and angiogenesis by sponging microRNA (miR)-429 in glioma cells (15). P73 antisense RNA 1T acts as a competing endogenous RNA to promote high mobility group proteins B1 (HMGB1) expression via sponging the miR-142 promoter, and thus functions as an oncogenic lncRNA by promoting proliferation and invasion in glioma cells (16).

Small ubiquitin-like modifier 1 pseudogene 3 (SUMO1P3), a newly identified IncRNA, serves an oncogenic role in bladder, breast and colon cancer, where it may be used as a potential prognostic biomarker (17-19). However, the effect of SUMO1P3 on glioma progression remains unknown. Thus, the present study investigated the expression pattern and function of SUMO1P3 in glioma and further analysed the downstream molecular signalling pathway. The present study demonstrated that SUMO1P3 may act as a tumour promoter in glioma, and may be used as a novel diagnostic biomarker and therapeutic target for glioma.

\section{Materials and methods}

Human tissues. Human brain tissue samples were collected from the Department of Neurosurgery at The Third Affiliated 
Hospital of Soochow University (Changzhou, China) during excision surgeries, between June 2015 and September 2017. A total of 12 glioma tissues (grade II, $n=4$; grade III, $n=4$ and grade $\mathrm{IV}, \mathrm{n}=4$ ) were obtained from patients with glioma (age range, 24-64 years; median age, 47 years) and histologically confirmed by three independent pathologists according to the 2016 WHO Classification of Tumors of the Central Nervous System (20). These patients were followed up by telephone every month for 12 months. A total of 10 tissue samples $(n=10)$ with no tumour complication were obtained from patients with cranial trauma (age range, 21-65 years; median age, 45 years), who served as the control. The tissues were immediately snap-frozen and stored in liquid nitrogen until subsequent experimentation. The present study was approved by the Research Ethics Board of the Third Affiliated Hospital of Soochow University and written informed consent was provided by patients or their guardians prior to the start of the study.

Cell lines and culture. A total of three glioma cell lines, including U87, LN229 and U251, and the human glial cell line, HEB, were purchased from The Cell Bank of Type Culture Collection of the Chinese Academy of Sciences. The U87 cell line used in the present study was authenticated by Shanghai VivaCell Biosciences Ltd., with STR profiling (http://www. vivacell.com.cn/Helps/STRAuthentication.html) as the U87 MG American Type Culture Collection (ATCC) version, which was a glioblastoma of unknown origin. Cells were cultured in Dulbecco's Modified Eagle's medium (DMEM; Gibco; Thermo Fisher Scientific, Inc.) with $10 \%$ foetal bovine serum (FBS; Gibco; Thermo Fisher Scientific, Inc.) at $37^{\circ} \mathrm{C}$ with $5 \% \mathrm{CO}_{2}$.

Cell transfection. Synthetic SUMO1P3 small interfering (si) RNA and a scrambled non-targeting siRNA as negative control siRNA (si-NC) were purchased from Shanghai GenePharma Co., Ltd. Cells were transfected using Lipofectamine ${ }^{\circledR} 2000$ transfection reagent (Invitrogen; Thermo Fisher Scientific, Inc.), according to the manufacturer's instructions. Briefly, $10 \mu \mathrm{l}$ siRNA $(20 \mu \mathrm{mol} / \mathrm{l})$ was mixed with $150 \mu \mathrm{l}$ Opti-Mem medium (Gibco; Thermo Fisher Scientific, Inc.) in one tube. $5 \mu$ Lipofectamine ${ }^{\circledR} 2000$ reagent was mixed with $150 \mu \mathrm{l}$ Opti-Mem medium in another tube. The contents of the two tubes were mixed and incubated at room temperature for $5 \mathrm{~min}$. The mixture was subsequently added to the cells in 6 -well plates and the plates were incubated at $37^{\circ} \mathrm{C}$. After $24 \mathrm{~h}$, the medium was replaced with DMEM with $10 \%$ FBS. The cells were collected for subsequent experimentation following $24 \mathrm{~h}$ of further culture. The sequences of these oligonucleotides were as follows: siSUMO1P3-302, 5'-GGCGUUCCAAUG AAUUCAUTT-3'; siSUMO1P3-877, 5'-CUUAAUUCAAGC UACUCUTT-3'; siSUMO1P3-946, 5'-GAUAACUGAUAA GGAGAGATT-3'; and si-NC, 5'-UUCUCCGAACGUGUC ACGUTT-3'. Cells were transfected using Lipofectamine ${ }^{\circledR T M}$ 2000 transfection reagent (Invitrogen; Thermo Fisher Scientific, Inc.), according to the manufacturer's instructions.

Reverse transcription-quantitative $(R T-q) P C R$. Total RNA was extracted from cells and human tissues using TRIzol ${ }^{\circledR}$ reagent (Invitrogen; Thermo Fisher Scientific, Inc.), according to the manufacturer's instructions. The purity and concentration of the RNA was evaluated with NanoDrop 2000 (Thermo Fisher Scientific, Inc.). Subsequently, $1 \mu \mathrm{g}$ RNA was used to synthesize cDNA using the PrimeScript ${ }^{\mathrm{TM}}$ RT Reagent kit (Takara Bio, Inc.) according to the manufacturer's instructions. qPCR was subsequently performed using SYBR Green PCR Master mix (Takara Bio, Inc.) on the ABI 7500 system (Applied Biosystems; Thermo Fisher Scientific, Inc.). The thermocycling conditions were as follows: $10 \mathrm{~min}$ at $95^{\circ} \mathrm{C}$, followed by 40 cycles for $10 \mathrm{sec}$ at $95^{\circ} \mathrm{C}$ and $40 \mathrm{sec}$ at $60^{\circ} \mathrm{C}$. GAPDH served as the endogenous control for SUMO1P3 and the relative gene expression was calculated using the $2^{-\Delta \Delta \mathrm{Cq}}$ method (21). The following primer sequences were used for qPCR: SUMO1P3 forward, 5'-ACTGGGAATGGAGGAAGA-3' and reverse, 5'-TGAGAAAGGATTGAGGGAAAAG-3'; GAPDH forward, 5'-GGAGCGAGATCCCTCCAAAAT-3' and reverse, 5'-GGCTGTTGTCATACTTCTCATGG-3'.

Western blotting. Proteins were extracted from cells using RIPA buffer (Beyotime Institute of Biotechnology) and the protein concentration was determined using BCA assays. Subsequently, proteins (30 $\mu \mathrm{g} /$ lane) were separated on $10 \%$ SDS-PAGE gels and then transferred onto PVDF membranes. After blocking with $10 \%$ non-fat milk for $1 \mathrm{~h}$ at room temperature, the membranes were incubated with antibodies against $\mathrm{N}$-cadherin (1:1,500; cat. no. 13116), E-cadherin $(1: 1,000$; cat. no. 14472), $\beta$-catenin $(1: 1,000$; cat. no. 8480), cyclin D1 (1:1,000; cat. no. 2978) and $\beta$-actin (1:2,500; cat. no. 3700$)$ overnight at $4^{\circ} \mathrm{C}$. Subsequently, the membranes were incubated with a secondary horseradish peroxidase (HRP)-labelled goat anti-mouse $\mathrm{IgG}$ (1:3,000; cat. no. A0216) or HRP-labelled goat anti-rabbit $\operatorname{IgG}(1: 3,000$; cat. no. A0208) for $1 \mathrm{~h}$ at room temperature. All the primary antibodies were purchased from Cell Signalling Technology, Inc., and the secondary antibodies were purchased from Beyotime Institute of Biotechnology. Protein bands were visualized using the ECL Advanced Western Blot Detection kit (Thermo Fisher Scientific, Inc.) on the Bio-Rad ChemiDoc ${ }^{\mathrm{TM}}$ Touch (Bio-Rad Laboratories, Inc.) and subsequently quantified using Image Lab software (version 5.2; Bio-Rad Laboratories, Inc.).

Wound healing assay. Transfected cells were seeded into 6 -well plates at a density of 50,000 cells/well. When the cell confluence reached $\sim 85 \%$, scratches were performed and the cells were incubated in serum-free medium. The plates were observed under a IX71 light microscope (magnification, x100; Olympus Corporation) at 0 and $48 \mathrm{~h}$ after the scratch, and the distance was measured using ImageJ software (National Institutes of Health).

Cell proliferation assay. Cell proliferation was assessed via the Cell Counting Kit-8 (CCK-8) assay (Beyotime Institute of Biotechnology) according to the manufacturer's instructions. Transfected cells were seeded into 96-well plates at a density of 3,000 cells/well. At the indicated time points $(0,24,48$ and $72 \mathrm{~h}$ ), $10 \mu \mathrm{l} \mathrm{CCK}-8$ regent was added into each well, and the plates were incubated at $37^{\circ} \mathrm{C}$ for $2 \mathrm{~h}$. The absorbance was measured at a wavelength of $450 \mathrm{~nm}$ using BioTek Elx800 (BioTek Instruments, Inc.). 
Migration and invasion assays. As previously described, Transwell plates were used to assess cell migration and invasion in vitro $(22,23)$. For cell invasion assays, the chambers (Corning, Inc.) were precoated with Matrigel (BD Biosciences) at $37^{\circ} \mathrm{C}$ for $5 \mathrm{~h}$. The chambers were observed in six randomly selected fields under a IX71 light microscope (magnification, x200; Olympus Corporation). The number of migrated cells in every field was counted.

Cell cycle analysis. Transfected cells were collected, fixed in $70 \%$ ethanol solution for $6 \mathrm{~h}$ at $4^{\circ} \mathrm{C}$. Cells were then washed with PBS and subsequently incubated with PI and RNase from a cell cycle and apoptosis analysis kit (cat. no. C1052; Beyotime Institute of Biotechnology) for $30 \mathrm{~min}$ at $37^{\circ} \mathrm{C}$ according to the manufacturer's instructions. The cell cycle was analysed on a Guava EasyCyte 6HT-2L flow cytometer (EMD Millipore), and assessed using the ModFit LT software (Version 3.1; Verity Software House, Inc.).

Statistical analysis. All experiments were performed independently in triplicates, and data were presented as the mean \pm standard error of the mean. Statistical analysis was performed using GraphPad Prism (version 8.3.0; GraphPad Software, Inc.) and the SPSS statistical software package (version 22.0; IBM Corp.). Unpaired Student's t-test was performed for comparisons between two groups. One-way ANOVA followed by Tukey's post-hoc test was used for multiple comparisons. Kaplan-Meier analysis and log-rank test were used for survival analysis of patients with glioma with low and high expression levels of SUMO1P3. P $<0.05$ was considered to indicate a statistically significant difference.

\section{Results}

SUMO1P3 expression is upregulated in glioma and high SUMO1P3 expression is associated with a poor prognosis. SUMO1P3 expression was assessed in 12 glioma and 10 control tissues. The clinical parameters of the tissue donors are described in Table I. As presented in Fig. 1A, SUMO1P3 expression was significantly higher in glioma tissues compared with in control tissues. The association between SUMO1P3 expression and glioma malignancy was also analysed. As presented in Fig. 1B, SUMO1P3 expression increased with increasing glioma grade. The present study further investigated whether SUMO1P3 was a prognostic factor for glioma. The median expression value of SUMO1P3 expression was used as a cut-off value to divide all 12 glioma patients into two groups. Patients with SUMO1P3 levels lower than the cut-off value were placed in the low expression group and patients with higher SUMO1P3 expression were assigned to the high expression group. Kaplan-Meier survival analysis indicated that high SUMO1P3 expression was associated with a poorer overall survival in patients with glioma compared with low SUMO1P3 expression (Fig. 1C). Furthermore, SUMO1P3 expression was assessed in 3 glioma cell lines. The results demonstrated that SUMO1P3 expression was upregulated in U251 and LN229 glioma cells compared with in HEB cells, however there was no significant difference between U87 and HEB cells (Fig. 1D). Thus, U251 and LN229 glioma cells were selected for subsequent experiments. Overall, the
Table I. Clinical parameters of tissue donors

\begin{tabular}{lcc}
\hline $\begin{array}{l}\text { Clinicopathological } \\
\text { parameters }\end{array}$ & Glioma $(\mathrm{n}=12)$ & $\begin{array}{c}\text { Control } \\
(\mathrm{n}=10)\end{array}$ \\
\hline Sex & & \\
Male & 6 & 5 \\
Female & 6 & 5 \\
Age, years & & \\
$<50$ & 6 & 5 \\
$\geq 50$ & 6 & 5 \\
WHO grade & & \\
II & 4 & NA \\
III & 4 & NA \\
IV & 4 & \\
Follow-up & & NA \\
Alive & 4 & NA \\
Dead & 8 & NA \\
Mean survival time, & $9.8 \pm 5.9$ & \\
months & &
\end{tabular}

The data of mean survival time are presented as mean survival time \pm standard error of the mean. WHO, World Health Organization; NA, not applicable.

present results suggested that SUMO1P3 may be a prognostic biomarker for glioma and may act as a tumour promoter in glioma.

SUMO1P3-knockdown suppresses cell proliferation and cell cycle in vitro. To investigate the function of SUMO1P3 in glioma, its expression was suppressed via transfection with siRNA in glioma cell lines. As presented in Fig. 2A and B, SUMO1P3 expression was significantly decreased following transfection with siSUMO1P3-302 in U251 and LN229 cells. The relative expression level in the siSUMO1P3-302 group was $30 \%$ of the si-NC group, thus siSUMO1P3-302 was selected for subsequent experimentation. The results of the cell proliferation assay demonstrated that SUMO1P3-knockdown significantly supressed U251 and LN229 cell proliferation (Fig. 2C). The influence of SUMO1P3 on cell cycle distribution was subsequently assessed. As presented in Figs. 2D and S1, SUMO1P3-knockdown resulted in the accumulation of cells in $\mathrm{G}_{1}$ phase, with 11.24 and $13.46 \%$ more cells in $\mathrm{G}_{1} / \mathrm{G}_{0}$ phase in the U251 and LN229 glioma cell lines compared with the si-NC groups. Meanwhile, there was a significant decrease in cells in $\mathrm{S}$ and $\mathrm{G}_{2} / \mathrm{M}$ phases in the siSUMO1P3-302 groups compared with cells in the si-NC groups. Collectively, the current results suggested that SUMO1P3-knockdown suppressed cell proliferation in glioma via $\mathrm{G}_{0} / \mathrm{G}_{1}$ cell cycle arrest.

SUMO1P3-knockdown represses cell migration and invasion in vitro. To assess the role of SUMO1P3 in cell migration and invasion, wound healing and Transwell assays were performed. The results of the wound healing assay demonstrated that SUMO1P3-knockdown significantly decreased the speed of wound closure in U251 and 

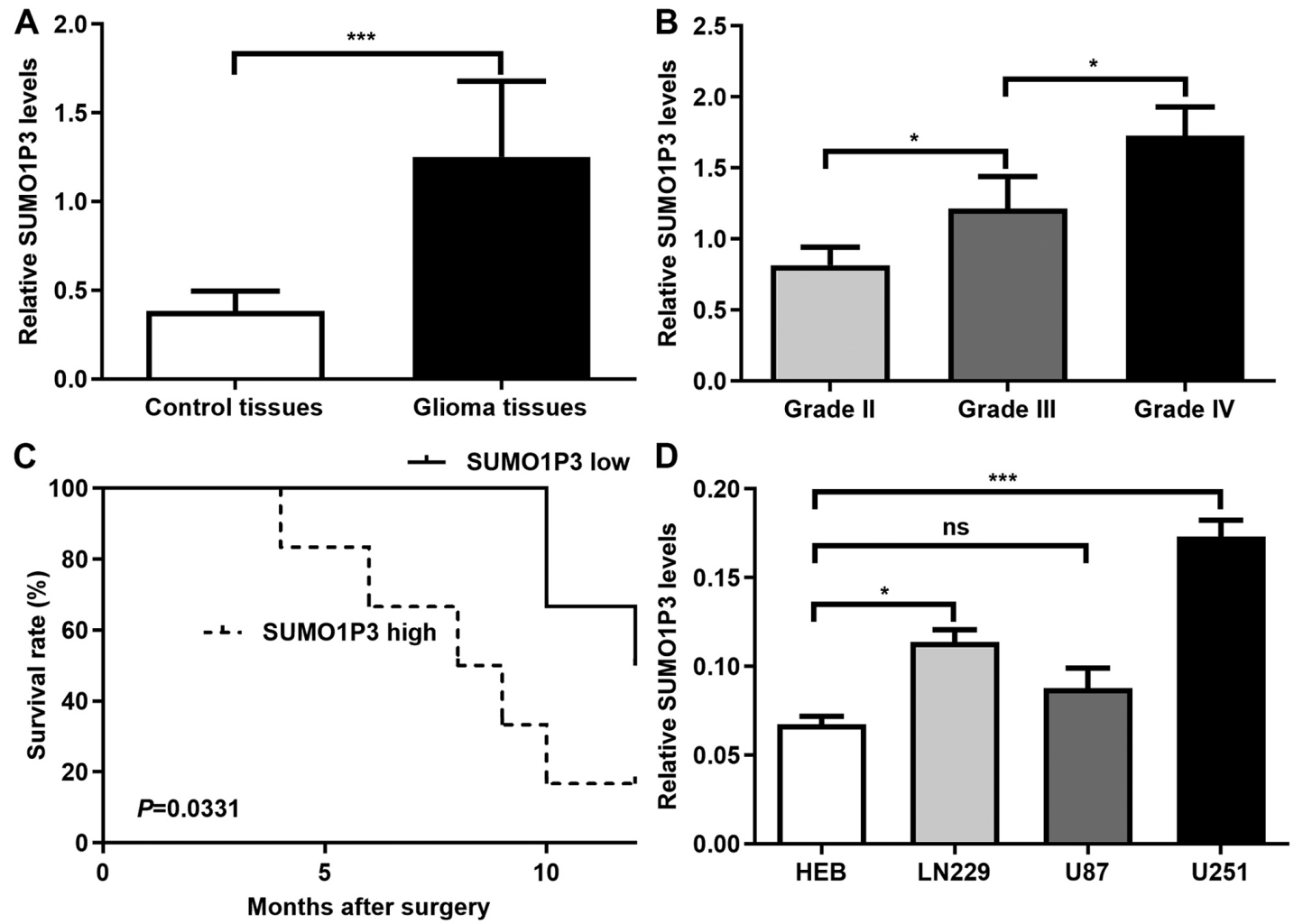

Figure 1. SUMO1P3 expression is upregulated in glioma. (A) Relative SUMO1P3 expression in control and glioma tissues. (B) Relative SUMO1P3 expression in glioma tissues with different grades. (C) Kaplan-Meier survival curves for patients with glioma with low and high expression levels of SUMO1P3. (D) Reverse transcription-quantitative PCR analysis was performed to detect relative SUMO1P3 expression in different cell lines. Data are presented as the mean \pm standard error of the mean. ${ }^{*} \mathrm{P}<0.05 ;{ }^{* * *} \mathrm{P}<0.001$. ns, not significant; SUMO1P3, small ubiquitin-like modifier 1 pseudogene 3.
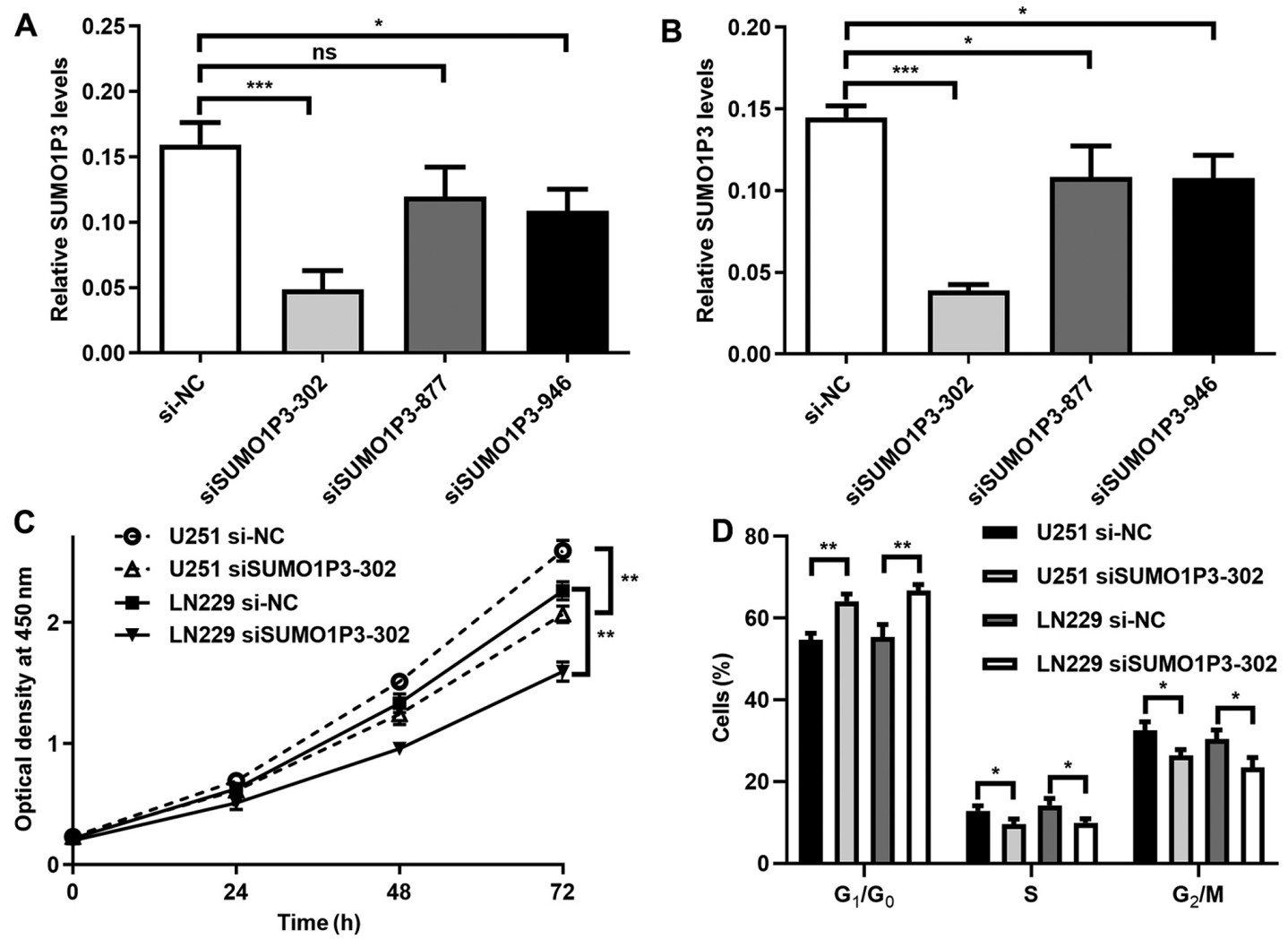

Figure 2. SUMO1P3-knockdown represses cell proliferation and cell cycle in vitro. Reverse transcription-quantitative PCR analysis was performed to detect relative SUMO1P3 expression in (A) U251 and (B) LN229 cells after siRNA transfection. Role of SUMO1P3-knockdown in (C) cell proliferation and (D) cell cycle. Data are presented as the mean \pm standard error of the mean. ${ }^{*} \mathrm{P}<0.05 ;{ }^{* * *} \mathrm{P}<0.01 ;{ }^{* * * *} \mathrm{P}<0.001$. ns, not significant; SUMO1P3, small ubiquitin-like modifier 1 pseudogene 3; siRNA, small interfering RNA; NC, negative control. 
A

B

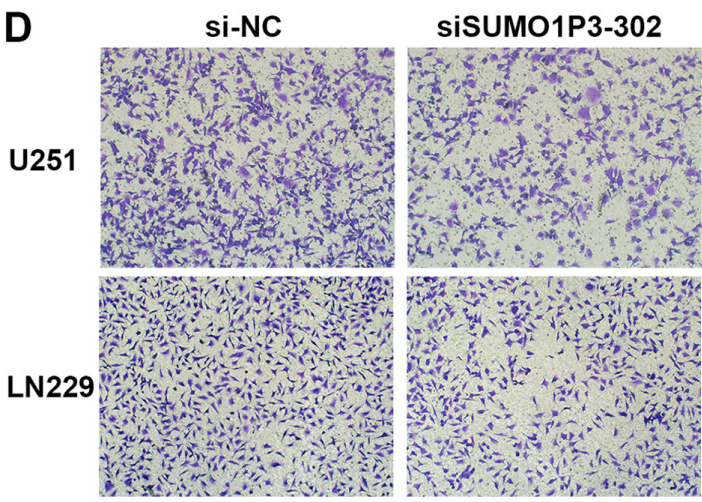

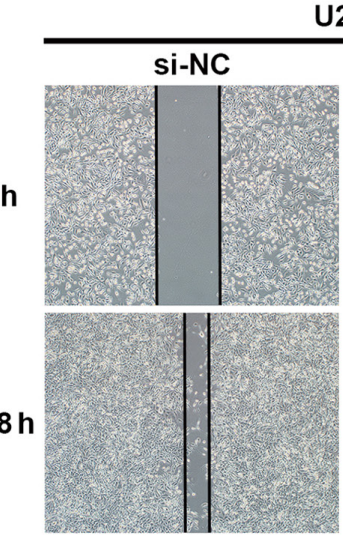

U251

$\mathrm{U} 251$

siSUM01P3-302
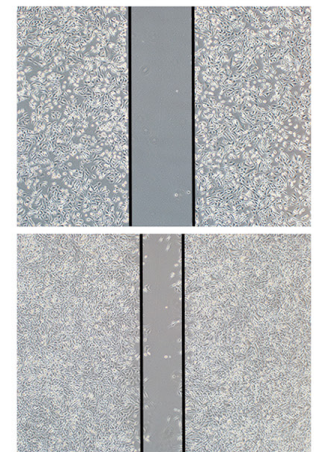

SiSUM01P3-302

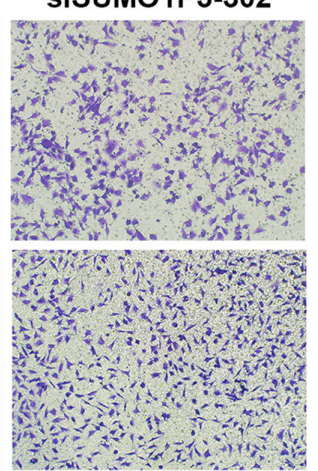

LN229

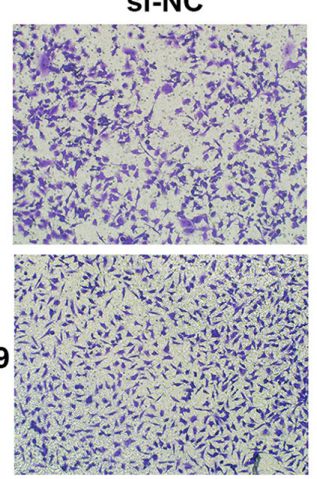

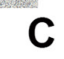

C
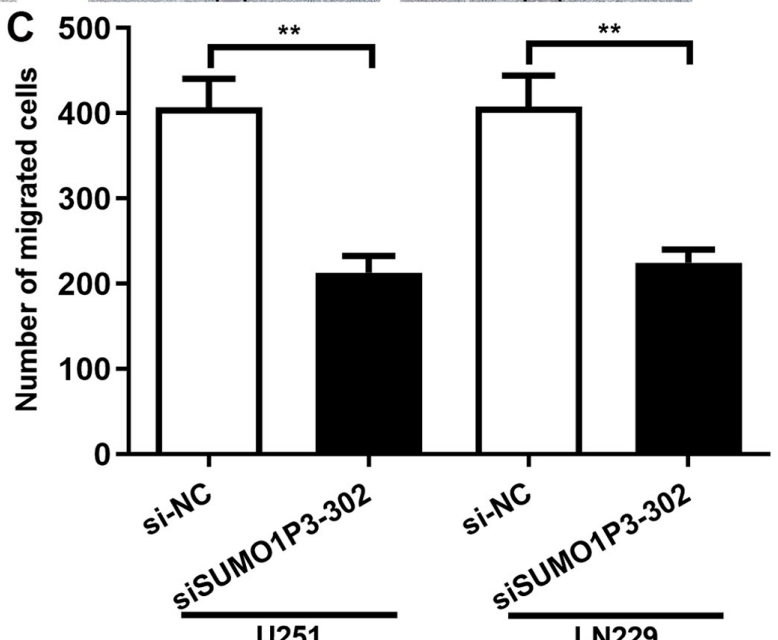

E

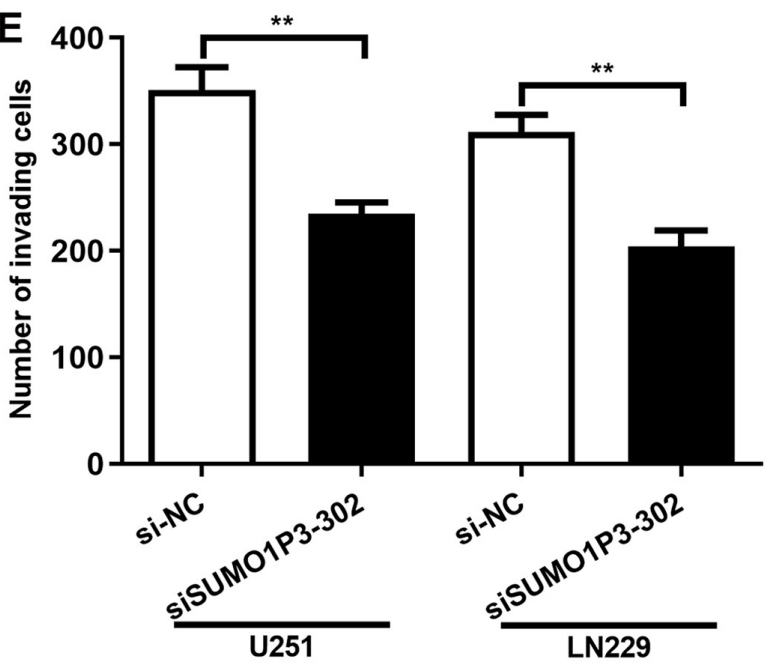

Figure 3. SUMO1P3-knockdown represses cell migration and invasion in vitro. (A) Representative results of the wound healing assay in SUMO1P3-knockdown cells (magnification, x100). (B) Representative results of the cell migration assay in SUMO1P3-knockdown cells (magnification, x200). (C) Statistical analysis for the results of the cell migration assay. (D) Representative results of the cell invasion assay in SUMO1P3-knockdown cells (magnification, x200), (E) Statistical analysis for the results of the cell invasion assay. Data are presented as the mean \pm standard error of the mean. ${ }^{* * *} \mathrm{P}<0.01$. SUMO1P3, small ubiquitin-like modifier 1 pseudogene 3; si, small interfering RNA; NC, negative control.

LN229 cells. Statistical analysis has been performed and the results showed that the relative wound closure percentage of U251 and LN229 cells at $48 \mathrm{~h}$ was 23.38 and $27.79 \%$ in the siSUMO1P3-302 group, respectively, and 44.87 and $39.21 \%$ in the si-NC group, respectively (Fig. $3 \mathrm{~A}$ ). The role of SUMO1P3 on cell migration and invasion was also investigated. The results of the Transwell assay demonstrated that the number of migrated U251 and LN229 cells in the
siSUMO1P3-302 group was 213 and 225 at $48 \mathrm{~h}$, significantly lower than the 407 and 408 cells in the si-NC group, respectively (Fig. 3B and C). In accordance with the results of cell migration, SUMO1P3-knockdown significantly supressed the invasive ability of U251 and LN229 cells (Fig. 3D and E). Overall, the present results suggested that SUMO1P3-knockdown supressed the migratory and invasive abilities of U251 and LN229 cells. 
A
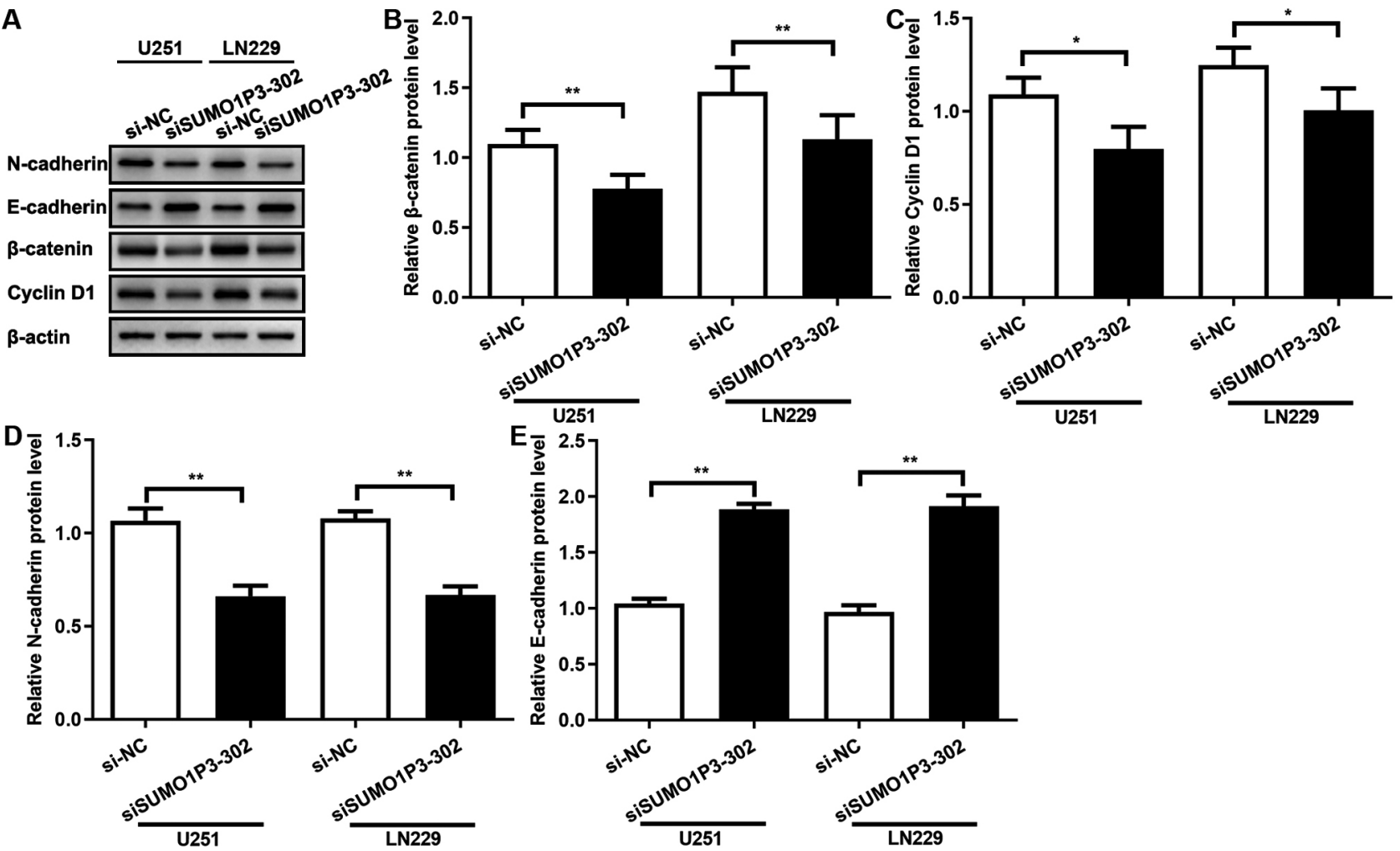

Figure 4. SUMO1P3 acts as a tumour promoter by regulating the expression levels of $\beta$-catenin, cyclin-D1, N-cadherin and E-cadherin. (A) Western blot analysis was performed to detect relative protein expression levels of N-cadherin, E-cadherin, $\beta$-catenin and cyclin-D1 in U251 and LN229 siRNA-transfected glioma cells. (B-E) Statistical analysis for the western blotting results of (B) $\beta$-catenin, (C) cyclin-D1, (D) N-cadherin and (E) E-cadherin. Data are presented as the mean \pm standard error of the mean. ${ }^{*} \mathrm{P}<0.05 ;{ }^{* *} \mathrm{P}<0.01$. SUMO1P3, small ubiquitin-like modifier 1 pseudogene 3; siRNA, small interfering RNA; NC, negative control.

SUMO1P3 acts as a tumour promoter via regulating the expression levels of $\beta$-catenin, cyclin-D1, $N$-cadherin and E-cadherin. The results of the present study demonstrated that SUMO1P3 acted as a tumour promoter in glioma cells by regulating cell proliferation, migration and invasion, whereas the underlying molecular mechanism remains unknown. $\beta$-catenin protein expression was assessed following transfection with siRNA, and the results demonstrated that SUMO1P3-knockdown significantly supressed $\beta$-catenin expression in both cell lines (Fig. 4A and B). Cyclin-D1 expression was also assessed, which is associated with the cell cycle and a downstream signalling protein of $\beta$-catenin (24). As presented in Fig. 4A and C, SUMO1P3-knockdown significantly supressed cyclin-D1 expression. The expression levels of proteins associated with migration and invasion were subsequently assessed. As presented in Fig. 4A, D and E, SUMO1P3-knockdown significantly supressed N-cadherin expression and significantly increased E-cadherin expression. Overall, the current results suggested that SUMO1P3 regulated cell proliferation, cell cycle, migration and invasion by regulating the expression levels of $\beta$-catenin, cyclin-D1, $\mathrm{N}$-cadherin and E-cadherin.

\section{Discussion}

Previous studies have reported that lncRNAs are aberrantly expressed in cancer and may serve a crucial role during tumour progression $(6,13,16,25)$. To the best of our knowledge,
Mei et al (26) has been the first to report that SUMO1P3 expression is upregulated in gastric cancer tissues and may serve as a potential biomarker for the diagnosis of gastric cancer. Since then, multiples studies have demonstrated the expression pattern and function of SUMO1P3 in different types of tumour. SUMO1P3 expression is upregulated in bladder cancer tissues, and SUMO1P3-knockdown inhibits cell proliferation and migration, while inducing apoptosis in bladder cancer cells (17). Additionally, SUMO1P3 expedites the malignant behaviours of colon cancer in vivo, such as growth, metastasis and angiogenesis, by regulating the expression levels of cyclin D1, vimentin, vascular endothelial growth factor A and E-cadherin (19). A recent study on breast cancer has suggested that SUMO1P3 acts as an oncogenic lncRNA by targeting miR-320a (18), identified as a tumour suppressor in different types of cancer, such as gastric cancer, non-small cell lung cancer and colorectal cancer (27-29). miR-320a supresses $\beta$-catenin expression by directly targeting the 3'-untranslated region of $\beta$-catenin mRNA in prostate cancer cells (30). In addition, SUMO1P3 promotes hepatocellular carcinoma progression through enhancing the $\mathrm{Wnt} / \beta$-catenin signalling pathway by sponging miR-320a (31). Thus, it is possible that SUMO1P3-knockdown represses $\beta$-catenin expression by targeting miR-320a in glioma.

Cyclin D1 is a downstream molecule of $\beta$-catenin (24). Cyclin D1 mediates the progression from $G_{1}$ to $S$ phase, thus slowing the proliferation of cancer cells (32). In the present study, SUMO1P3-knockdown supressed proliferation in 
glioma cells probably via cell cycle arrest caused by suppression of cyclin D1. $\beta$-catenin and E-cadherin are epithelial cell markers, and $\mathrm{N}$-cadherin is a mesenchymal phenotype marker during the EMT process (33). Increasing evidence suggests that an E-cadherin to $\mathrm{N}$-cadherin shift is mainly involved in EMT and serves a key role in glioma progression and invasion (34). According to the present results, it may be speculated that SUMO1P3-knockdown may supress cell migration and invasion by regulating the expression levels of $\beta$-catenin, $\mathrm{N}$-cadherin and E-cadherin. However, the current study presents some limitations. Firstly, only 12 glioma tissues and 10 control tissues were used in the present study. The number of patients was relatively small, therefore further studies using more human tissues should be performed to confirm the relationship between SUMO1P3 expression and glioma malignancy. Furthermore, control tissues were obtained from patients with severe acute traumatic brain injury during a needed surgery, thus theses tissues were not healthy tissues. Despite consent for the collection of tissues was provided by the patients or their guardians, it would have been unethical for healthy tissue to be used. A recent study has reported that SUMO1P3 promotes glioma cell proliferation, migration, and invasion via the $\mathrm{Wnt} / \beta$-catenin pathway (35). The results and conclusions are consistent with the present study, except for the expression levels of SUMO1P3 in U87 glioma cell line. Lou et al (35) demonstrated high SUMO1P3 expression in U87 cells, whereas the present study identified there was no significant difference between U87 and HEB cells. Since there are two versions of U87 cell line, one is the original glioblastoma cell line established in the University of Uppsala (https://web.expasy.org/cellosaurus/CVCL_GP63) and the other is the U87 MG ATCC version, which is most probably a glioblastoma but whose origin is unknown (https://web.expasy.org/cellosaurus/CVCL_0022), there are differences in the behaviours of these cell lines. The present study confirmed the U87 cell line used was the ATCC version by STR profiling. Regarding Lou et al have not clarified the version of U87 cell line used, it is possible that they used the original glioblastoma cell line established in the University of Uppsala.

In conclusion, the present study revealed the expression pattern of SUMO1P3 and its tumorigenic function in glioma tissues and cells. SUMO1P3-knockdown was demonstrated to suppress cell proliferation, cell cycle, migration and invasion by regulating the expression levels of $\beta$-catenin, cyclin-D1, $\mathrm{N}$-cadherin and E-cadherin in U251 and LN229 glioma cells. Overall, the current results suggest that SUMO1P3 may act as a novel diagnostic biomarker and therapeutic target for glioma.

\section{Acknowledgements}

Not applicable.

\section{Funding}

The present study was funded by Changzhou Municipal Commissions of Health and Family Planning Major Scientific and Technological Project (grant no. ZD201620), Changzhou Municipal Commission of Health and Family Planning Youth Talent Scientific and Technological Project (grant no. QN201807) and Funding from Young Talent Development Plan of Changzhou Health Commission (grant no. CZQM2020042).

\section{Availability of data and materials}

The datasets used and/or analysed during the current study are available from the corresponding author on reasonable request.

\section{Authors' contributions}

DD and JC collaborated to design the study, performed the cell cycle assay, analysed the data, drafted the initial manuscript and confirmed the authenticity of the raw data. YM performed RT-qPCR, western blotting and the wound healing assays. LX performed cell proliferation, migration and invasion assays. NS acquired and analysed the data of patients, collected the human tissues and helped in revising the paper. All authors read and approved the final manuscript.

\section{Ethics approval and consent to participate}

The present study was approved by the Research Ethics Board of the Third Affiliated Hospital of Soochow University (grant no. 2020031; Changzhou, China). Written informed consent was provided by all patients or their guardians.

\section{Patient consent for publication}

Not applicable.

\section{Competing interests}

The authors declare that they have no competing interests.

\section{References}

1. Siegel RL, Millaer KD and Jemal A: Cancer statistics, 2017. CA Cancer J Clin 67: 7-30, 2017.

2. Bielle F: Building diagnoses with four layers: WHO 2016 classification of CNS tumors. Rev Neurol (Paris) 172: 253-255, 2016.

3. Boussiotis VA and Charest A: Immunotherapies for malignant glioma. Oncogene 37: 1121-1141, 2018.

4. Van Meir EG, Hadjipanayis CG, Norden AD, Shu HK, Wen PY and Olson JJ: Exciting new advances in neuro-oncology: The avenue to a cure for malignant glioma. CA Cancer J Clin 60: 166-193, 2010.

5. Stupp R, Taillibert S, Kanner A, Read W, Steinberg D, Lhermitte B, Toms S, Idbaih A, Ahluwalia MS, Fink K, et al: Effect of tumor-treating fields plus maintenance temozolomide vs maintenance temozolomide alone on survival in patients with glioblastoma: A randomized clinical trial. JAMA 318: 2306-2316, 2017.

6. Bhan A, Soleimani M and Mandal SS: Long noncoding RNA and cancer: A new paradigm. Cancer Res 77: 3965-3981, 2017.

7. Wang JZ, Xu CL, Wu H and Shen SJ: LncRNA SNHG12 promotes cell growth and inhibits cell apoptosis in colorectal cancer cells. Braz J Med Biol Res 50: e6079, 2017.

8. Wang O, Yang F, Liu Y, Lv L, Ma R, Chen C, Wang J, Tan Q, Cheng Y, Xia E, et al: C-MYC-induced upregulation of lncRNA SNHG12 regulates cell proliferation, apoptosis and migration in triple-negative breast cancer. Am J Transl Res 9: 533-545, 2017.

9. Wang P, Chen D, Ma H and Li Y: LncRNA SNHG12 contributes to multidrug resistance through activating the MAPK/Slug pathway by sponging miR-181a in non-small cell lung cancer. Oncotarget 8: 84086-84101, 2017. 
10. Zhang $\mathrm{H}$ and $\mathrm{Lu} \mathrm{W}$ : LncRNA SNHG12 regulates gastric cancer progression by acting as a molecular sponge of miR320. Mol Med Rep 17: 2743-2749, 2018

11. Gordon MA, Babbs B, Cochrane DR, Bitler BG and Richer JK: The long non-coding RNA MALAT1 promotes ovarian cancer progression by regulating RBFOX2-mediated alternative splicing. Mol Carcinog 58: 196-205, 2019.

12. Xu Y, Zhang X, Hu X, Zhou W, Zhang P, Zhang J, Yang S and Liu Y: The effects of IncRNA MALAT1 on proliferation, invasion and migration in colorectal cancer through regulating SOX9. Mol Med 24: 52, 2018.

13. Ye Y, Zhang F, Chen Q, Huang Z and Li M: LncRNA MALAT1 modified progression of clear cell kidney carcinoma (KIRC) by regulation of miR-194-5p/ACVR2B signaling. Mol Carcinog 58: 279-292, 2019.

14. Jia L, Tian Y, Chen Y and Zhang G: The silencing of LncRNA-H19 decreases chemoresistance of human glioma cells to temozolomide by suppressing epithelial-mesenchymal transition via the Wnt/3-Catenin pathway. Onco Targets Therapy 11: 313-321, 2018

15. Cheng Z, Li Z, Ma K, Li X, Tian N, Duan J, Xiao X and Wang Y: Long non-coding RNA XIST promotes glioma tumorigenicity and angiogenesis by acting as a molecular sponge of miR-429. J Cancer 8: 4106-4116, 2017.

16. Zhang R, Jin $\mathrm{H}$ and Lou F: The long non-coding RNA TP73-AS1 interacted with miR-142 to modulate brain glioma growth through HMGB1/RAGE pathway. J Cell Biochem 119: 3007-3016, 2018.

17. Zhan Y, Liu Y, Wang C, Lin J, Chen M, Chen X, Zhuang C, Liu L, $\mathrm{Xu} \mathrm{W}$, Zhou Q, et al: Increased expression of SUMO1P3 predicts poor prognosis and promotes tumor growth and metastasis in bladder cancer. Oncotarget 7: 16038-16048, 2016.

18. Liu J, Song Z, Feng C, Lu Y, Zhou Y, Lin Y and Dong C: The long non-coding RNA SUMO1P3 facilitates breast cancer progression by negatively regulating miR-320a. Am J Trans Res 9: 5594-5602, 2017.

19. Zhang LM, Wang P, Liu XM and Zhang YJ: LncRNA SUMO1P3 drives colon cancer growth, metastasis and angiogenesis. Am J Transl Res 9: 5461-5472, 2017.

20. Louis DN, Perry A, Reifenberger G, von Deimling A, Figarella-Branger D, Cavenee WK, Ohgaki H, Wiestler OD, Kleihues P and Ellison DW: The 2016 World Health Organization Classification of tumors of the central nervous system: A summary. Acta Neuropathol 131: 803-820, 2016.

21. Livak KJ and Schmittgen TD: Analysis of relative gene expression data using real-time quantitative PCR and the 2(-Delta Delta C(T)) method. Methods 25: 402-408, 2001.

22. Deng D, Luo K, Liu H, Nie X, Xue L, Wang R, Xu Y, Cui J, Shao N and Zhi F: p62 acts as an oncogene and is targeted by miR-124-3p in glioma. Cancer Cell Int 19: 280, 2019.

23. Deng D, Xue L, Shao N, Qu H, Wang Q, Wang S, Xia X, Yang Y and Zhi F: miR-137 acts as a tumor suppressor in astrocytoma by targeting RASGRF1. Tumour Biol 37: 3331-3340, 2016.
24. Chattopadhyay S, Chaklader M and Law S: Aberrant Wnt signaling pathway in the hematopoietic stem/progenitor compartment in experimental leukemic animal. J Cell Commun Signal 13: 39-52, 2019

25. Sun Y, Zheng ZP, Li H, Zhang HQ and Ma FQ: ANRIL is associated with the survival rate of patients with colorectal cancer, and affects cell migration and invasion in vitro. Mol Med Rep 14: 1714-1720, 2016

26. Mei D, Song H, Wang K, Lou Y, Sun W, Liu Z, Ding X and Guo J: Up-regulation of SUMO1 pseudogene 3 (SUMO1P3) in gastric cancer and its clinical association. Med Oncol 30: 709, 2013.

27. Ge X, Cui H, Zhou Y, Yin D, Feng Y, Xin Q, Xu X, Liu W, Liu S and Zhang Q: miR-320a modulates cell growth and chemosensitivity via regulating ADAM10 in gastric cancer. Mol Med Rep 16: 9664-9670, 2017.

28. Zhao W, Sun Q, Yu Z, Mao S, Jin Y, Li J, Jiang Z, Zhang Y, Chen M, Chen $\mathrm{P}$, et al: MiR-320a-3p/ELF3 axis regulates cell metastasis and invasion in non-small cell lung cancer via PI3K/Akt pathway. Gene 670: 31-37, 2018.

29. Zhao H, Dong T, Zhou H, Wang L, Huang A, Feng B, Quan Y, Jin R, Zhang W, Sun J, et al: miR-320a suppresses colorectal cancer progression by targeting Racl. Carcinogenesis 35: 886-895, 2014

30. Hsieh IS, Chang KC, Tsai YT, Ke JY, Lu PJ, Lee KH, Yeh SD, Hong TM and Chen YL: MicroRNA-320 suppresses the stem cell-like characteristics of prostate cancer cells by downregulating the Wnt/beta-catenin signaling pathway. Carcinogenesis 34: 530-538, 2013

31. Wu S, Chen S, Lin $\mathrm{N}$ and Yang J: Long non-coding RNA SUMO1P3 promotes hepatocellular carcinoma progression through activating $\mathrm{Wnt} / \beta$-catenin signalling pathway by targeting miR-320a. J Cell Mol Med 24: 3108-3116, 2020.

32. Fu M, Wang C, Li Z, Sakamaki T and Pestell RG: Minireview: Cyclin D1: Normal and abnormal functions. Endocrinology 145: 5439-5447, 2004.

33. Tam WL and Weinberg RA: The epigenetics of epithelialmesenchymal plasticity in cancer. Nat Med 19: 1438-1449, 2013.

34. Noh MG, Oh SJ, Ahn EJ, Kim YJ, Jung TY, Jung S, Kim KK, Lee JH, Lee KH and Moon KS: Prognostic significance of E-cadherin and N-cadherin expression in Gliomas. BMC cancer 17: 583, 2017.

35. Lou JY, Luo J, Yang SC, Ding GF, Liao W, Zhou RX, Qiu CZ and Chen JM: Long non-coding RNA SUMO1P3 promotes glioma progression via the Wnt $/ \beta$-catenin pathway. Eur Rev Med Pharmacol Sci 24: 9571-9580, 2020.

c) (i) $\Theta$ This work is licensed under a Creative Commons EY NO NO Attribution-NonCommercial-NoDerivatives 4.0 International (CC BY-NC-ND 4.0) License. 